à pouvoir garantir la texture voulue dans le fromage fini, il est capital que l'acidité se développe progressivement, et que les tauxlimites précis indiqués pour chaque stade ne soient pas dépassés. Le temps nécessaire sera tel qu'il devra permettre une acidité de $0,5 \%$ à la mise en moule s'il s'agit d'un fromage acidophilus, acidité qui devrait atteindre $0,9 \%$ pour un caillé de cheddar ordinaire lors de la mise sous presse. Si le lait est un lait a normal (par exemple, si l'une des vaches productrices est atteinte de mammite), le développement de l'acidité peut être arrêté à un degré plus avancé avec l'emploi de $B$. acidophilus comme levain qu'avec celui d'un levain commercial habituel (Str. cremoris et Str. lactis).

\title{
QUELQUES DONNÉES SUR LA TENEUR DU SANG DES VACHES LAITIËRS EN CHOLESTÉROL ET EN PHOSPHATIDES
}

\author{
par
}

\section{A. DOULKIN et S. HELMAN}

(Institut de Laiterie de Molochnaïa, près de Vologda, U. R. S. S.)

Le rôle des lipoïdes en physiologie et en pathologie a été l'objet de nombreuses investigations expérimentales. Sans vouloir donner une revue complète de tous les travaux (nous renvoyons les lecteurs à la revue de Smorodinzeff (1929), qui contient le résumé des 260 travaux), nous nous permettons de fixer quelques points.

Le rôle du cholestérol dans le métabolisme doit être immense, car nous le trouvons dans chaque cellule. Un homme reçoit journellement dans sa ration de 0,039 à 1,406 gr. de cholestérol. Néanmoins nous devons conclure, que l'organisme peut synthétiser le cholestérol, car dans l'œil extirpé (lapin, bœuf), le maximum de sa teneur en cholestérol n'est atteint que six heures environ après l'extirpation. Chez les omnivores, la teneur du sang en cholestérol démontre une faible augmentation dans les quelques heures qui suivent l'ingestion de cholestérol avec la ration. Chez les herbivores, cela ne se produit qu'après deux ou trois jours. Pendant un jeûne prolongé, quand l'organisme perd un tiers de son poids et presque toute sa graisse, sa teneur en cholestérol ne tombe pas au-dessous d'un certain minimum. C'est seulement vers la mort qu'on observe une chute brusque.

Comme l'a démontré R. Brinkman (1920), e'est le rapport qui existe entre la teneur en cholestérol et celle des phosphatides qui contrôle la teneur de la cellule en eau. RuBner, d'autre part, a démontré, dans ses expériences, que c'est le métabolisme de l'eau, pendant la période de croissance, qui a un rôle éminent. Des expé- 
riences ont démontré qu'un supplément de cholestérol au milieu nutritif augmente la vitesse de croissance chez les infusoires. La lécithine, d'autre part, entrave la croissance. Le cholestérol favorise de même la croissance des tumeurs. En parlant du rôle du cholestérol dans la croissance, il ne faut pas oublier que le facteur antirachitique, la vitamine $\mathrm{D}$, est un dérivé du cholestérol.

Chez les animaux maintenus dans des locaux pourvus d'une faible ventilation, on observe un accroissement de la teneur du sang en cholestérol. Il y a des observations qui montrent, que la teneur des muscles en cholestérol est proportionnelle à la durée du travail, ce qui peut être intéressant dans l'étude des bêtes de travail. Il a été aussi démontré que le cholestérol stimulait le développement du poil, ce qui peut être intéressant dans l'oviculture. L'hypercholestérémie diminue la vitalité des jeunes cellules; c'est pourquoi chez les diabétiques, on observe une prédisposition pour les dermatites et une lente cicatrisation des blessures. Le cholestérol peut être considéré comme un antagoniste des substances provoquant l'hémolyse, comme la lécithine, le venin de serpent et la digitanine. Avec la digitanine, le cholestérol donne une substance cristalline, laquelle peut même être utilisée pour sa détermination quantitative.

C'est le mérite du Professeur Ch. Porcher (1922) d'avoir signalé la relation possible entre les lipides du sang et la production du beurre ehez la vache laitière. J. MARCQ et A. Leroy (1930) ont cru avoir prouvé que c'était la teneur du sang en lipides totaux qui pouvait servir d'indice de la valeur beurrière des bovidés.

Roodzant (1931), se basant sur les expériences de Meigs, Blatherwick et CARY (1919) et de SJolema (1923), regarde les phosphatides (la lécithine) comme la substance à partir de laquelle la glande mammaire synthétise la graisse du lait. Ses propres expériences le conduisent à nier la corrélation entre la richesse du lait en matières grasses et la teneur du sang en lipides totaux, posée par Marcq et Leroy. D'autre part, il croit avoir donné la preuve que la teneur du sang en lécithine est en corrélation étroite $(r=0,65$ $\pm 0,093)$ avec la richesse beurrière du lait.

P. Masaers, de son côté, pense (1931) avoir prouvé que « seul, le

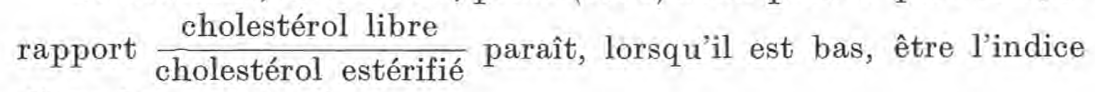
d'une bonne beurrière ".

Ces contradictions ont contraint MARCQ à répéter ses expériences. Sa dernière publication (en collaboration avec le $\mathrm{D}^{\mathrm{r}}$ DEvurst ; 1933) montre qu'il met en doute ses données antérieures, autant que les données du $\mathrm{D}^{\mathrm{r}}$ Roodzant, car il ne peut constater dans ses recherches postérieures ni la corrélation entre la richesse du lait avec les lipides totaux du sang, ni avec la teneur du sang en lécithine. 
Il faut remarquer que la technique de ROozDANT se distinguait de celle du Professeur MARCQ. Le premier opérait avec la teneur du serum sanguin en lécithine, tandis que le dernier, avec la teneur du sang total (plasma + coagulum).

Se basant sur ses propres recherches aussi bien que sur les recherches d'autres investigateurs (MAYNARD, HARrison et McCAY) (1931), J. MarcQ souligne la grande variabilité de la teneur du sang en lipides (totaux, et divers), sous l'influence des conditions ambiantes : l'alimentation, la période de lactation, l'âge, la race, etc. Il pense pour cela qu'il faut recommencer les recherehes dans une exploitation " comprenant un nombre suffisant de bêtes adultes, dont les mises bas seraient assez voisines; et sur lesquelles on échelonnerait régulièrement les prélèvements sanguins ").

Comme la teneur du lait en matière grasse de la même vache est des plus variable d'un échantillon à un autre, il pense que " le rendement total par période de lactation doit être pris comme indice d'une vache bonne beurrière et non simplement la teneur au jour du prélèvement d'un échantillon ".

Dans notre ouvrage, nous avions en conséquence pour but, d'établir les relations qui existent entre la teneur du sang en cholestérol et en lécithine, d'une part, et la production quantitative et qualitative des vaches laitières, d'autre part. Nous voulions rechercher l'influence de certaines conditions d'alimentation et d'entretien (conditions hygiéniques) sur les mêmes indices.

\section{Matériel et Méthode}

Nos investigations ont eu lieu sur 14 vaches de la race locale, qui étaient en cours d'expériences d'alimentation dans la vacherie expérimentale de l'Institut de Laiterie Molochnaïa (près de Vologda, U. R. S. S.).

Un des groupes de vaches subissait l'expérience sur l'influence de l'ionisation de l'air, un autre celle d'alimentation des vaches laitières avec des algues marines. Les vaches étaient spécialement choisies pour ces expériences parmi le troupeau de la ferme de l'Institut (contenant plus de 1.000 vaches) et étaient toutes à peu près à la même période de lactation. Les variations observées dans la teneur du sang des vaches différentes prises aux mêmes stades, doivent par conséquent être imputées à l'individualité propre des divers animaux.

Le prélèvement des échantillons de sang se faisait dans la veine jugulaire à la quantité de $20-25 \mathrm{~cm}^{3}$, avant la traite de midi. Les prélèvements ont eu lieu en janvier, en mars et en juin 1933. Nous avons déterminé la teneur du sang en cholestérol par la méthode d'Engelhardt et Smirnova et de la lécithine (= le phosphore lipémique) suivant la méthode de Bloor. 
L'échantillon de sang prélevé aseptiquement était mis à coaguler dans un thermostat à la to de $38^{\prime \prime} \mathrm{C}$. Le sérum sanguin était ensuite centrifugé à l'aide d'une centrifuge électrique et était soumis à l'analyse. Pour les dosages colorimétriques, nous nous servions du colorimètre Dubosc.

Nous suivions la technique de Roodzant et non celle du Professeur MARCQ en faisant nos dosages dans le sérum sanguin et non dans le sang total, car nous pensons que c'est principalement le sérum qui est le siège du métabolisme lipique des cellules et non les éléments formés.

\section{Relation entre la teneur du sang en cholestérol et la production du lait en $\mathbf{3 0 0}$ jours de lactation}

Les échantillons de sang furent pris le 13 et le 22 janvier.

TABLEAU I.

\begin{tabular}{|c|c|c|c|c|}
\hline $\mathrm{N}^{\circ}$ & Nom de la vache & $\begin{array}{l}\text { Teneur du sang } \\
\text { en cholestérol }\end{array}$ & $\begin{array}{l}\text { Rendement } \\
\text { de lait } \\
\text { en } 300 \text { jours }\end{array}$ & $\begin{array}{c}\text { Date } \\
\text { de l'analyse } \\
\text { du sang }\end{array}$ \\
\hline 1. & Voltrane $\ldots \ldots \ldots \ldots \ldots \ldots$ & 289 & 1778 & $13 / \mathrm{I}$ \\
\hline 2. & Isola $\ldots \ldots \ldots \ldots \ldots \ldots$ & 278 & 1879 & $22 / \mathrm{I}$ \\
\hline 3. & Microna $\ldots \ldots \ldots \ldots \ldots \ldots \ldots$ & 259 & 2727 & $22 / \mathrm{I}$ \\
\hline 4. & Catoda...$\ldots \ldots \ldots \ldots \ldots$ & 258 & 2854 & $22 / \mathrm{I}$ \\
\hline 5. & Electrida ............... & 235 & 2553 & $22 / \mathrm{I}$ \\
\hline 6. & Dosa $\ldots \ldots \ldots \ldots \ldots \ldots$ & 217 & 2823 & $13 / \mathrm{I}$ \\
\hline 7. & Roentgena $\ldots \ldots \ldots \ldots \ldots \ldots$ & 186 & 2492 & $13 / \mathrm{I}$ \\
\hline 8. & Ionina $\ldots \ldots \ldots \ldots \ldots \ldots \ldots$ & 131 & 3426 & 13/I \\
\hline
\end{tabular}

On peut remarquer dans ce tableau (tableau I), que les vaches à plus grand rendement en lait ont une teneur du sang en cholestérol moins grande que celle des vaches moins bonnes laitières. Cela peut être confirmé en calculant le coefficient de corrélation $\mathrm{r}=-0,87 \pm$ 0,087 .

Teneur du sang en phosphore lipémique ( $=$ en lécithine) et Richesse du lait au jour du prélèvement de l'échantillon en matières grasses.

Ire série. Les vaches étaient soumises à l'expérience d'alimentation avec des algues marines. 
TABLEAU II.

\begin{tabular}{|c|c|c|c|c|c|c|c|c|c|}
\hline \multicolumn{5}{|c|}{ Groupe en expérience } & \multicolumn{5}{|c|}{ Groupe-témoin } \\
\hline No & $\begin{array}{c}\text { Nom } \\
\text { de la vache }\end{array}$ & $\begin{array}{l}\text { P. lipé- } \\
\text { mique }\end{array}$ & $\begin{array}{l}\text { Graisse } \\
\text { du lait }\end{array}$ & Date & $N^{\circ}$ & $\begin{array}{l}\text { Nom } \\
\text { de la vache }\end{array}$ & $\begin{array}{l}\text { P. lipé- } \\
\text { mique }\end{array}$ & $\begin{array}{l}\text { Graisse } \\
\text { du lait }\end{array}$ & Date \\
\hline 1. & Roentgena & 18,65 & 5,5 & 14/VI & 1. & Isola...... & 14,15 & 4,5 & $14 / V_{I}$ \\
\hline 2. & Magneta... . & 10,35 & 5,2 & $17 / V I$ & 2. & Catoda... & 12,42 & 3,9 & $17 / \mathrm{VI}$ \\
\hline 3. & Ionina $\ldots$ & 8,55 & 3,6 & $17 / \mathrm{VI}$ & 3. & Dosa .... & 8,33 & 3,2 & $14 / V I$ \\
\hline
\end{tabular}

IIe Série. Les vaches étaient soumises à l'expérience de l'ionisation de l'air de l'étable.

TABLEAU III.

\begin{tabular}{|c|c|c|c|c|c|c|c|c|}
\hline \multicolumn{5}{|c|}{ Groupe en expérience } & \multicolumn{4}{|c|}{ Groupe-témoin } \\
\hline No & $\begin{array}{l}\text { Nom de } \\
\text { la vache }\end{array}$ & $\begin{array}{l}\text { P. lipé. } \\
\text { mique }\end{array}$ & $\begin{array}{l}\text { Graisse } \\
\text { du lait }\end{array}$ & Date & $\mathrm{N}^{0}$ & $\begin{array}{l}\text { Nom } \\
\text { de la vache }\end{array}$ & $\begin{array}{l}\text { P. lipé- } \\
\text { mique }\end{array}$ & $\begin{array}{l}\text { Graisse } \\
\text { du lait }\end{array}$ \\
\hline 1. 1 & Iskra ... & 11,55 & 4,06 & 14/III & 1. & Roentgena & 14,1 & 4,90 \\
\hline 2. I & Magneta & 10,27 & 4,34 & n & 2. & Isola ..... & 13,74 & 4,08 \\
\hline 3. & Argenta. & 9,2 & 3,98 & n & 3. & Catoda ... & 11,66 & 3,50 \\
\hline 4. & Dosa ... & 6,3 & 3,50 & ” & 4. & Ionina ... & 10,28 & 3,60 \\
\hline & & & & & 5. & Dosa ..... & 8,15 & 3,85 \\
\hline & & & & $\therefore$ & 6. & Electrida.. & 6,13 & 3,47 \\
\hline
\end{tabular}

Les deux séries montrent, avec assez d'ensemble, la corrélation qui existe entre le taux du phosphore lipémique (la lécithine) du sérum sanguin et la richesse du lait en matières grasses. Le coefficient de corrélation est positif et plus grand que la triple erreur moyenne fortuite

$$
r=+0,85 \pm 0,124
$$

Nos expériences, quoique exécutées sur un nombre d'animaux fort restreint, semblent done confirmer les données précitées de ROODZANT.

\section{Variation des indices lipémiques sous l'influence des conditions ambiantes}

A. La saison.

Les échantillons du sang furent pris chez huit vaches en hiver. Ces vaches étaient au régime de stabulation. Leur alimentation consistait en foin de prairie (de 6,2 à 12,4 kg. par vache et par jour), 
en son de froment (de 0,95 à $5,8 \mathrm{~kg}$. par vache et par jour), en tourteaux de lin (de 0 à $3,31 \mathrm{~kg}$.), et en farine d'avoine (de 0 à $1,0 \mathrm{~kg}$.).

Des échantillons de sang furent prélevés chez six de ces mêmes vaches, en été.

Le tableau IV montre que le taux du cholestérol est beaucoup plus élevé en hiver que pendant les mois d'été chez les mêmes vaches.

Un changement du taux du cholestérol dans la même direction a été observé par les collaborateurs de 1'Institut de Biologie expérimentale de Moscou dans leur investigation sur la corrélation entre la composition du sang et du lait des vaches (AvDEEva et al. 1932). Leurs matériaux montrent que le taux du cholestérol du sang des vaches de la race Yaroslave changea de la façon suivante du régime de stabulation à celui du pâturage.

TABLEAU IV.

\begin{tabular}{|c|c|c|c|c|c|c|c|}
\hline \multirow[b]{2}{*}{ No } & \multicolumn{4}{|c|}{ Hiver } & \multicolumn{3}{|c|}{ Eté } \\
\hline & Nom de la vache & $\begin{array}{l}\text { Choles- } \\
\text { térol }\end{array}$ & P. lip. & $\frac{\text { Chol. }}{\text { P. lip. }}$ & $\begin{array}{l}\text { Choles- } \\
\text { térol }\end{array}$ & P. lip. & $\frac{\text { Chol. }}{\text { P. lip. }}$ \\
\hline 1. & Ionina $\ldots \ldots \ldots \ldots$ & 131 & 12,00 & 10,9 & 50,5 & 8,55 & 5,9 \\
\hline 2. & Roentgena ........ & 186 & 9,45 & 19,7 & 124,0 & 18,65 & 6,65 \\
\hline 3. & Dosa ............. & 217 & 7,70 & 26,8 & 185 & 8,33 & 22,2 \\
\hline 4. & Voltana .......... & 289 & 9,45 & 30,7 & - & - & - \\
\hline 5. & Catoda ........... & 258 & 10,92 & 23,6 & 157 & 12,42 & 12,7 \\
\hline 6. & Isola $\ldots \ldots \ldots \ldots \ldots$ & 278 & 12,73 & 21,8 & 175,0 & 14,75 & 11,85 \\
\hline 7. & Microna............ & 259 & - & - & - & - & - \\
\hline 8. & Electrida ........... & 235 & 6,77 & 34,7 & 102 & 5,5 & 18,5 \\
\hline \multirow{2}{*}{\multicolumn{2}{|c|}{$\begin{array}{l}\text { M. pour } 6 \text { vaches ...... } \\
\text { M. pour } 8 \text { vaches........ }\end{array}$}} & 217,5 & 9,61 & 22,9 & 138,6 & 11,81 & 12,85 \\
\hline & & 231,5 & & & & & \\
\hline
\end{tabular}

Nous pensons que la conclusion des dits auteurs, tendant à imputer cette chute du taux du cholestérol du sang à l'alimentation des vaches pendant l'hiver de l'année 1931 (qui précédait la saison

TABLEAU V.

\begin{tabular}{c|c|c|c|c}
\hline \multirow{2}{*}{ Année } & Mois & $\begin{array}{c}\text { Nombre } \\
\text { de vaches }\end{array}$ & $\begin{array}{c}\text { Cholestérol } \\
\text { du sang }\end{array}$ & Régime \\
\cline { 3 - 5 } 1930 & mars-avril & 17 & 301,4 & de stabulation d'hiver \\
1931 & juin-juillet & 24 & 173,7 & d'été au pâturage \\
\hline
\end{tabular}

du pâturage de l'été 1931 des dites vaches), n'est pas valable. Les 
faits expérimentaux ont démontré que l'alimentation, même chez les herbivores, produit son influence sur la teneur du sang en cholestérol, au plus tard dans les trois jours. Dans l'expérience des auteurs, les vaches étaient au régime du pâturage au moins un mois avant la prise des échantillons du sang pour l'analyse. L'influence de l'alimentation de l'hiver écoulé (si elle existait !) devait être effacée depuis longtemps sous l'influence de l'alimentation suffisante au pâturage. D'autre part, nous savons que l'alimentation insuffisante, même le jeûne absolu, ne diminuent que d'une manière insignifiante le taux du cholestérol du sang. Nous pensons pour cela que c'était l'influence légère de l'insolation directe, l'influence de l'air pur du pâturage et du fourrage vert succulent qui contribuèrent le plus à la diminution du taux du cholestérol dans l'organisme des vaches au régime du pâturage.

Des observations (voir SMonoDINzeFF, 1929) montrent que l'alimentation avee du sucre et avec des pommes de terre favorise la diminution du taux du cholestérol dans l'organisme du chien et que, d'un autre côté, une alimentation copieuse des chiens arec de la viande et de la graisse contribue à l'augmentation de la cholestérémie.

Taux des indices lipémiques du sang sous l'influence de l'alimentation avec des algues (Laminaria-saccharina L.) séchées.

Les expériences de l'alimentation des vaches laitières avec des algues marines (Laminaria saccharina L.) eurent lieu pendant l'été (mois de juin) de l'année 1933. Les vaches en expérience recevaient une ration de $28 \mathrm{~kg}$. d'algues et de 2,1 à $5,5 \mathrm{~kg}$. de son de froment. Les vaches du groupe témoin recevaient $8 \mathrm{~kg}$. de foin et de 2,1 à 5,5 de son de froment.

TABLEAU VI.

\begin{tabular}{|c|c|c|c|c|c|c|c|c|c|}
\hline \multicolumn{5}{|c|}{ Alimentation avec des algues } & \multicolumn{5}{|c|}{ Groupe-témoin } \\
\hline No & $\begin{array}{c}\text { Nom } \\
\text { de la vache }\end{array}$ & $\begin{array}{l}\text { Choles. } \\
\text { térol } \\
\text { du sang }\end{array}$ & $\begin{array}{l}\text { P. lipé- } \\
\text { mique }\end{array}$ & $\frac{\text { Cholest. }}{\text { P. lip. }}$ & No & $\begin{array}{l}\text { Nom de } \\
\text { la vache }\end{array}$ & $\begin{array}{l}\text { Choles- } \\
\text { térol }\end{array}$ & $\begin{array}{l}\text { P. lipé- } \\
\text { mique }\end{array}$ & $\frac{\text { Cholest. }}{\text { P. lip. }}$ \\
\hline 1. & Roentgena & 124 & 18,65 & 6,65 & 1. & Isola ... & 175,12 & 14,75 & 11,8 \\
\hline 2. & Magneta... & 151 & 14,65 & 10,3 & 2. & Catoda.. & 157,0 & 12,42 & 12,7 \\
\hline 3. & Ampera.... & 80 & 11,75 & 6,8 & 3. & Dosa..... . & 185,0 & 8,33 & 22,2 \\
\hline 4. & Ionina ... & 50 & 8,55 & 5,8 & 4. & $\longrightarrow$ & - & - & - \\
\hline & M. . . . & 101,25 & 13,4 & 7,5 & & & 188,3 & 12,51 & 15,0 \\
\hline
\end{tabular}

Nous voyons que la substitution du foin de la ration par les 
algues marines eut, pour résultat, une chute considérable de la teneur du sang en cholestérol. Le taux du phosphore lipémique changea peu. Nous avons, en conséquence, un doublement de l'indice cholestérol

phosphore lipémique

\section{Régime de pâturage}

Le régime de pâturage a les mêmes conséquences. Les vaches Electrida et Quarzina montrèrent en juin respectivement 102 et 91,75 gr. de cholestérol dans le sérum sanguin, étant au pâturage, ce qui est un taux inférieur à celui présenté par les vaches tenues en même temps au régime de stabulation (groupe témoin de l'expérience de l'alimentation avec des algues).

Nous pouvons en conséquence tirer la conclusion que l'influence des algues marines sur l'organisme de la vache est analogue à celui du fourrage vert du pâturage. Signalons que le Professeur J. DoLGicH, qui conduisait ces expériences, aboutit à la même conclusion en analysant la question d'un tout autre point de vue. (Sous presse.)

\section{Influence de 1'ionisation de 1'air de 1'étable}

$\mathrm{Au}$ mois de mars, des échantillons de sang furent prélevés chez quatre vaches, qui étaient soumises à l'expérience sur l'influence de l'ionisation de l'air de l'étable sur la digestibilité de la ration (Dolgich et Perov, 1933). Les résultats furent les suivants : (voir tableau VII).

Si on compare les données du tableau VII avec celles du tableau I - prélèvement des échantillons de sang au mois de janvier - on peut voir une chute de la teneur moyenne du sérum sanguin sous l'influence de l'ionisation :

$$
231,6-165,6=66,0 .
$$

TABLEAU VII.

\begin{tabular}{|c|c|c|c|c|c|}
\hline No & $\begin{array}{l}\text { Nom } \\
\text { de la vache }\end{array}$ & $\begin{array}{l}\text { Choles- } \\
\text { térol } \\
\text { du sérum }\end{array}$ & $\begin{array}{l}\text { Phosphore } \\
\text { lipémique }\end{array}$ & $\begin{array}{c}\text { Relation } \\
\text { cholest./P. lip. }\end{array}$ & Alimentation \\
\hline 1. & Iskra ...... & 153,20 & 11,55 & 13,2 & \multirow{4}{*}{$\begin{array}{l}\text { foin : } 7,7 \text { aे } 8,7 \mathrm{~kg} \text {. } \\
\text { son : } 1,0 \text { à } 2,5 \mathrm{~kg} \text {. } \\
\text { tourteaux : } 1,0 \text { à } 2,5 \mathrm{~kg} \text {. }\end{array}$} \\
\hline 2. & Magneta... . & 153,25 & 10,27 & 14,9 & \\
\hline 3. & Dosa ..... & 149,00 & 6,29 & 22,9 & \\
\hline 4. & Argenta... & 207,00 & . 9,20 & 22,5 & \\
\hline & M. . . . & 165,60 & 9,40 & 17,6 & \\
\hline
\end{tabular}

Comme on peut le voir, l'influence de l'ionisation de l'air de 
l'étable est de la même sorte que l'influence de l'été. (Comparez les données du tableau IV.) L'air ionisé a la même influence stimulante sur le métabolisme qui aboutit à la plus grande diminution du taux du cholestérol dans l'organisme que le régime du pâturage d'été.

\section{Relation entre la teneur du sang en cholestérol et en phosphore lipémique}

Si nous opposons la teneur du sérum sanguin en cholestérol à sa teneur en phosphore lipémique, nous notons les résultats suivants :

Tableat VIII.

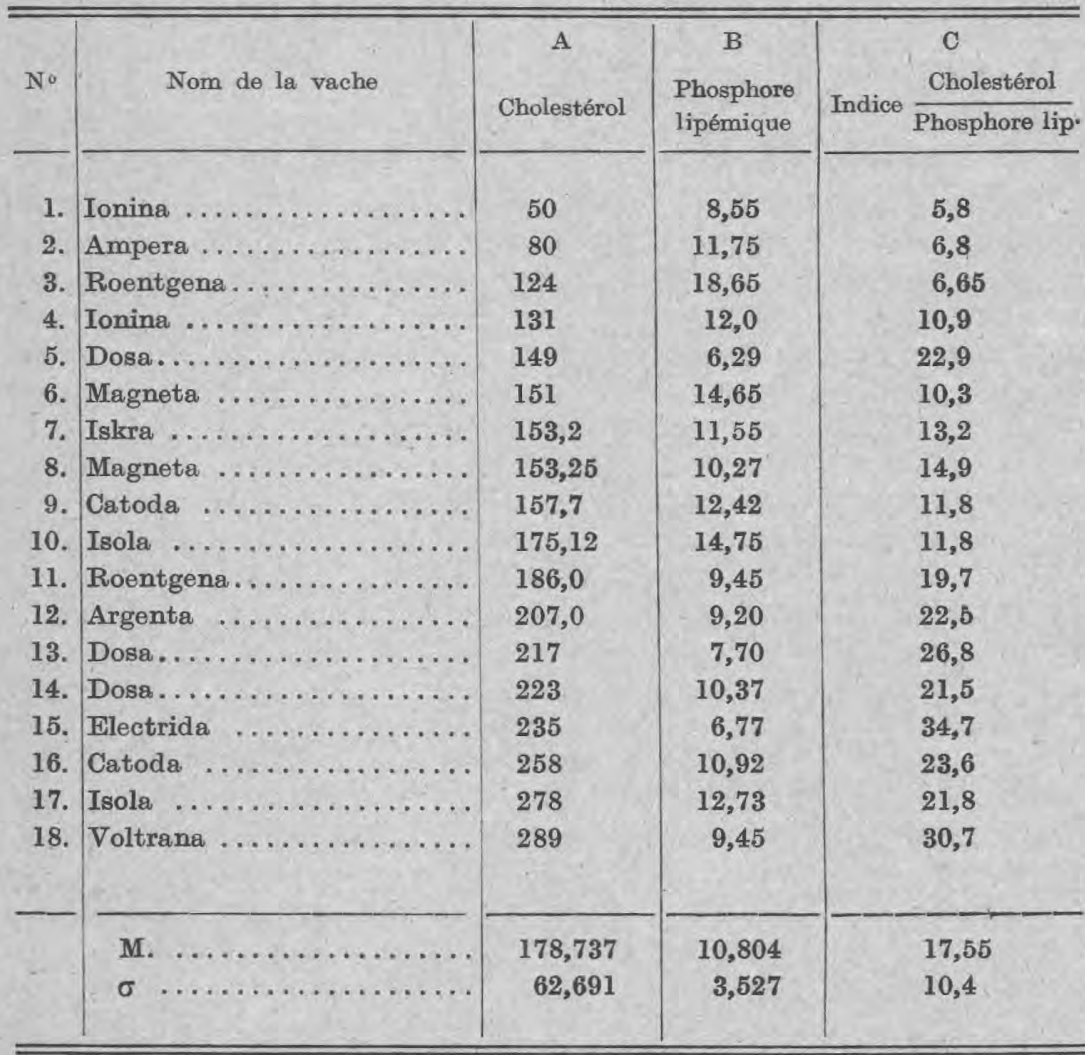

$$
\begin{array}{ll}
\mathrm{r}=-0,06 \pm 0,39 & \mathrm{r}=0,84 \pm 0,067 \\
\mathrm{~A}-\mathrm{B} & \mathrm{A}-\mathrm{C}
\end{array}
$$

Nous pouvons en conclure : $1^{\circ}$ que la variation de la teneur $d u$ sérum en cholestérol et la variation en phosphore lipémique sont indépendantes : $\mathrm{r}=-0,06 \pm 0,39$, et $2^{\circ} q u$ 'il existe une corrélation positive entre l'indice $\frac{\text { cholestérol }}{\text { phosphore lipémique }}$ et la teneur en cholestérol :
$\mathrm{r}=0,84$. 


\section{RÉSUME DES RÉSULTATS}

$1^{\circ}$ Il y a, entre la teneur en cholestérol du sérum sanguin d'une vache et sa production laitière quantitative, une haute corrélation négative (proportionnalité inverse) $(\mathrm{r}=-0,87 \pm 0,087)$.

$2^{\circ}$ Il y a une haute corrélation positive (proportionnalité directe) entre la teneur du sérum sanguin en lécithine (= phosphore lipémique) et la richesse du lait de la vache en matière grasse au temps du prélèvement de l'échantillon $(\mathrm{r}=0,85 \pm 0,124)$.

$3^{\circ}$ Le taux du cholestérol est en hiver plus haut qu'en été.

$4^{\circ}$ L'alimentation peut aroir une influence sur la teneur du sérum en cholestérol. Il est positivement prouvé que l'alimentation avec des algues marines (Laminaria saccharata) abaisse le taux du cholestérol.

50 L'ionisation de l'air amène elle aussi une chute du taux du cholestérol.

$6^{\circ}$ La même influence est constatée pour le régime de pâturage.

\section{DISCUSSION DES RÉSULTATS}

Toutes nos eonclusions ne peuvent être considérées que comme très préliminaires, car elles ont été faites après expériences sur un nombre fort restreint d'animaux. Néanmoins, nous pensons que nos résultats montrent que le problème, surtout celui de l'évaluation des propriétés beurrières d'une vache (d'une génisse et d'un taureau) par l'analyse de son sang, mérite une très grande considération et nos données doivent pousser à l'exécution de nouvelles expériences pour résoudre la question.

Deux mots sur la discordance de nos données avec celles du Professeur MardQ (1933). Comme nous l'avons écrit, eet auteur nie la dépendance existant entre la lécithine du sang et la richesse beurrière du lait de la vache. Nos expériences, en accord avec celles du $\mathrm{D}^{\mathrm{r}}$ Roodzant, semblent, au contraire, affirmer cette corrélation. La technique des expérimentateurs belges, il est vrai, diffère de la nôtre (comme de celle du Dr RooDZaNT) : ils font l'analyse du sang total (coagulum + sérum), tandis que nous recherchions la teneur du sérum seul, ce que nous considérons plus rationnel (voir plus haut). Le Professeur MARCQ pense que la teneur du lait en matière grasse étant soumise à des variations très grandes, même d'une traite à l'autre, au cours de la même journée, c'est le rendement total par période de lactation qui doit servir comme indice beurrier d'une vache et non la richesse beurrière du lait dans une certaíne période. Nous, de notre part, pensons que la richesse du lait ( $\%$ de matière grasse) est un élément indépendant et digne d'être étudié séparément de la production totale de matière grasse par lactation. Si la teneur du sérum sanguin en lécithine varie de période à période, de 
même que la richesse du lait de la même vache (il ne pourrait en être autrement si l'on admet que la richesse du lait dépend de la teneur du sérum en lécithine), cela ne montre, à la rigueur, que l'insuffisance de l'analyse d'un seul échantillon de sang d'une vache pour établir une caractéristique exacte de ses propriétés laitières. Il faudrait agir là comme dans l'évaluation de la richesse moyenne en matière grasse du lait d'une vache par lactation, en prenant un certain nombre d'échantillons.

En conclusion, nous voulons remercier le Professeur D. KIsSLowsky (chaire de zootechnie), qui nous proposa ce sujet de travail, le Professeur J. DoLGIOH (chaire d'alimentation du bétail), qui nous donna la possibilité d'utiliser les vaches qui étaient soumises à ses expériences, et le Dr L. LAPInsky (chef du laboratoire de biochimie), qui nous donna les possibilités de faire les analyses dans son laboratoire et qui eut la grande amabilité de nous donner des conseils éclairés au cours de notre travail.

\section{BIBLIOGRAPHIE}

[1] Avdeiva M., Borissenko E., Ivanova E., Mrssineva M., Provatorova M., SAvrtsch N. Studien über sekretion der Milchdrüse, Korrelation zwischen Blut- und Milchzusammensetzung. Journal de Biologie (texte russe et résumé allemand). Vol. I, fasc. 1-2, 1932, p. 4-40.

[2] BLOoR. 1929. Determination of phosphates in blood.Journ.Biol. Ohem. Vol. 36, 1929.

[3] Brinkman. 1920. Cité ehez Smorodinzeff, 1929.

[4] DoLgroH, 1933. L'ionisation et l'alimentation. Problemi Zhivotnovodstva. No 1 , p. 96-101 (russe).

[5] Engelihardt et Smirnova. Cité dans le livre de Balackovsky, 1932. Micro. analyse du sang et son importance clinique. Moscou, p. 317.

[6] Maroq et Lerox. 1930. Le Taux butyreux sanguin des reproducteurs bovins et ses rapports éventuels avec la valeur d'élevage. (Extrait des travaux du Congrès de Liége, p. 1-51.)

[7] Maroq et Devurst. 1933. - Contribution à l'étude de la valeur des lipides du sang en tant qu'indice de l'aptitude beurrière. (Extrait du Bull. de l'Institut agronomique de Gembloux. Tome II, n² 2, p. 100-126.)

[8] Masafers P. 1931. Contribution à l'étude de la lipémie de la vache. Il nuovo Ezcolani, vol. 36, p. 377-390. (Cité d'après le Recueil de Méd. Vétérinaire, CVIII, p. 498.

[9] Maynard L. A., HaRrisson E. S. and McCay C.M. 1931. 'he changes in the total fatty acids, phospholipid fatty acids and cholesterol of the blood during the lactation eycle. Journ. of Biological Chemistry, vol. XCII, $n^{\circ} 2$, p. 263-272.

[10] Meigs, Blatherwick and Cary. 1919. Contribution to the physiology of phosphorus and calcium metabolism as related to milk secretion. Jour. Biol. Chem., vol. 37, p. 1-75.

[11] Poncher Ch. 1922. Rapport au Congrès National de Laiterie à Rennes, octobre 1922. (Cité dans Le Lait, vol, 5, p. 11, 1925.) 
[12] Poroher Oh. et Maynard L. 1930. La graisse du sang et la graisse du lait pendant la lactation. Le Lait, vol. 10, p. 601-613 et 765-782.

[13] Roodzant. 1931. Onderzockingen betreffende de correlatie tussehen het vetgehalte van de Melk en het lipoïdenhalt van het bloed. Dissert. Utrecht.

[14] SjoLlima. 1923. Voeding an Melkproductie. Culture.

[15] SMorodtnzerry. 1929. Le cholestérol et son importance en physiologie et en pathologie. "Ouspecloie biologuitschesloi Chimii ", vol. VII, p. 104-135 (russe).

\section{LA MODIFICATION DE LA COMIPOSITION DU LAIT A L'ÉPOQUE DE LA PAISSON}

\section{par ZOLTAN CSUKAS}

Docteur vétérinaire et docteur agr., professeur adjoint d'université.

La science recherche depuis longtemps déjà dans quelle. mesure la paisson influence la composition du lait, et particulièrement sa teneur en graisse. Si la modification de la eomposition n'atteignait que des dixièmes de pourcentage, on pourrait trouver l'explication dans les conditions vitales variant quotidiennement et dont l'importance théorique et pratique est restreinte. Selon la littérature, cependant, la teneur en graisse du lait, à l'époque en question, peut tomber au-dessous de $2 \%$ ou monter au-dessus de $4,5 \%$, même dans une moyenne "d'étable».

L'observation des conditions contradictoires complique la = eomparaison des données écrites (HITTOHER et GRIMMER, BÜNGER et Altmann, Kirsten, Olmmanns, Sommerfeld, Stegmann, Thikötter, Amschler, Chrzaszoz, Cambell, Hills, Regsdale et Turner, Brody, Drakeley et White, Popoff, Housten et HaLE, etc.). Les données américaines, danoises, anglaises et, en partie, allemandes ont été obtenues sous des climats très variés, ce qui suffit à expliquer les résultats contradictoires. Dans les expériences d'étable (où les autres facteurs ont été éliminés), la corrélation néga tive entre la température ascendante et la teneur en graisse a aussi été constatée de façon unanime. Le contraire, cependant, a été observé dans les expériences de pâturage où le rôle alternatif de la variation de température ne s'est montré dans aucune direction.

Au cours de mes expériences, en 1932 et 1933, j'ai examiné dans les semaines précédant la pousse de l'herbe et pendant le mois suivant celle-ci, le lait de 140 vaches provenant de six exploitations agricoles différentes. En voici le résultat :

Dans les premières semaines de la paisson, la teneur en graisse du lait et en extrait sec dégraissé, change ainsi que la flore microbienne du pis. La cause de l'importance et du sens de la modification doit être cherchée dans les influences extérieures. 